\title{
Coupling of Two Superconductors through a Ferromagnet: Evidence for a $\pi$ Junction
}

\author{
V. V. Ryazanov, ${ }^{1}$ V. A. Oboznov, ${ }^{1}$ A. Yu. Rusanov, ${ }^{1}$ A. V. Veretennikov, ${ }^{1}$ A. A. Golubov, ${ }^{2}$ and J. Aarts ${ }^{3}$ \\ ${ }^{1}$ Institute of Solid State Physics, Russian Academy of Sciences, Chernogolovka, 142432, Russia \\ ${ }^{2}$ University of Twente, P.O. Box 217, 7500 AE Enschede, The Netherlands \\ ${ }^{3}$ Kamerlingh Onnes Laboratory, Leiden University, P.O. Box 9504, 2300 RA Leiden, The Netherlands
}

(Received 24 August 2000)

\begin{abstract}
We report measurements of the temperature dependence of the critical current, $I_{c}$, in Josephson junctions consisting of conventional superconducting banks of $\mathrm{Nb}$ and a weakly ferromagnetic interlayer of a $\mathrm{Cu}_{x} \mathrm{Ni}_{1-x}$ alloy, with $x$ around 0.5 . With decreasing temperature $I_{c}$ generally increases, but for specific thicknesses of the ferromagnetic interlayer, a maximum is found followed by a strong decrease down to zero, after which $I_{c}$ rises again. Such a sharp cusp can be explained only by assuming that the junction changes from a 0 -phase state at high temperatures to a $\pi$ phase state at low temperatures.
\end{abstract}

DOI: $10.1103 /$ PhysRevLett.86.2427

Almost all of the presently known superconductors contain conventional Cooper pairs, two electrons with opposite spin and momentum $(+\mathbf{k} \uparrow,-\mathbf{k} \downarrow)$. Such a system is described by an isotropic excitation gap or an order parameter. The exceptions are found notably in high $T_{c}$ superconductors, and in some organic superconductors and heavy fermion systems, in which the exact pairing mechanism is not yet fully understood. Still, it is surprising that of many possible ways to form a pair, so few are actually realized. For instance, it is not imperative that the net momentum of the pair is zero. It was predicted long ago by Larkin and Ovchinnikov [1] and by Fulde and Ferrel [2] that pairing still can occur when the electron energies and momenta at the Fermi energy are different for the two spin directions, for instance as the result of an exchange field in magnetic superconductors. The "LOFF" state is qualitatively different from the zero-momentum state: it is spatially inhomogeneous and the order parameter contains nodes where the phase changes by $\pi$. It was never observed in bulk materials, but below we present evidence that it can be induced in a weak ferromagnet (F) sandwiched between two superconductors (S). Such a SFS junction can yield a phase shift of $\pi$ between the superconducting banks, as was also predicted [3-5]. The $\pi$ state offers new ways for studying the coexistence of superconductivity and magnetism and may also be important for superconducting electronics, e.g., in quantum computing: several schemes for the necessary qubits (quantum two-level systems) rely on phase shifts of $\pi$ in a superconducting network $[6,7]$.

The spatial variation of the superconducting order parameter in the ferromagnet arises as a response of the Cooper pair to the energy difference between the two spin directions. The electron with the energetically favorable spin increases its momentum by $Q \propto E_{\text {ex }} / v_{F}$, where $E_{\text {ex }}$ is the exchange energy and $v_{F}$ is the Fermi velocity, while the other electron decreases its momentum by $Q$. Since the original momentum of each electron can be positive or negative, the total pair momentum inside the ferromagnet is $2 Q$ or $-2 Q$. Combination of the two possibilities
PACS numbers: 74.50.+r, 74.80.Dm

leads to an oscillating order parameter $\psi(z)$ in the junction along the direction normal to the SF interfaces: $\psi(z) \propto$ $\cos (2 Q z)[8,9]$. The same picture applies in the diffusive limit. Now the oscillation is superimposed on the decay of the order parameter due to pair breaking by impurities in the presence of the exchange field. In the regime $E_{\text {ex }} \gg k_{B} T$, the decay length $\xi_{F 1}$ is given by $\left(\hbar D / E_{\text {ex }}\right)^{1 / 2}$, where $D$ is the electron diffusion coefficient in the ferromagnet, while the oscillation period $2 \pi \xi_{F 2}$ is equal to $2 \pi\left(\hbar D / E_{\mathrm{ex}}\right)^{1 / 2}$. Because of the oscillations, different signs of the order parameter can occur at the two banks when the $F$-layer thickness $d_{F}$ is of the order of half a period. This is the so-called $\pi$-phase state, which competes for existence with the ordinary 0 -phase state. Figure 1a shows a Ginzburg-Landau free-energy calculation consisting of negative condensation energy and positive gradient energy for either state in the $F$ layer. The $\pi$ phase is more favorable in the range $d_{F} /\left(2 \pi \xi_{F 2}\right)$ between 0.4 and 0.8 . Figure $1 \mathrm{~b}$ shows the behavior of $\psi(z)$ in the $F$ layer below and above $d_{F, \mathrm{cr}}$. The crossover from the 0 phase to the $\pi$ phase state should manifest itself in an anomalous thickness dependence both of the superconducting transition temperature $T_{c}$ of the junction $[10,11]$ and of the critical current $I_{c}$ [4]. Experiments on $T_{c}\left(d_{F}\right)$ have been performed in systems such as $\mathrm{Nb} / \mathrm{Gd}[12], \mathrm{Nb} / \mathrm{Fe}[13], \mathrm{V} / \mathrm{Fe}$ [14], and $\mathrm{Pb} / \mathrm{Fe}$ [15] but the results are not conclusive. Especially, it was shown that also in bilayer systems (no coupling) $T_{c}\left(d_{F}\right)$ can behave in an anomalous fashion [15].

Our approach is to induce the crossover as a function of temperature, not of thickness, and to use a unique signature of the junction $I_{c}$ : according to the Josephson relation $I_{s}=I_{c} \sin (\phi)$, with $\phi$ the phase difference across the junction, biasing with $\phi=\pi$ should lead to a negative current response upon a small increase of the phase. In other words, $I_{c}$ becomes negative. A change of state from 0 to $\pi$ will lead to a zero crossing of $I_{c}$, and if only the absolute value of the current is measured, a sharp cusp will be observed. The condition for having the temperature as a parameter is $k_{B} T \approx E_{\mathrm{ex}}$. The exchange field and the temperature then are equally important and the behavior of 

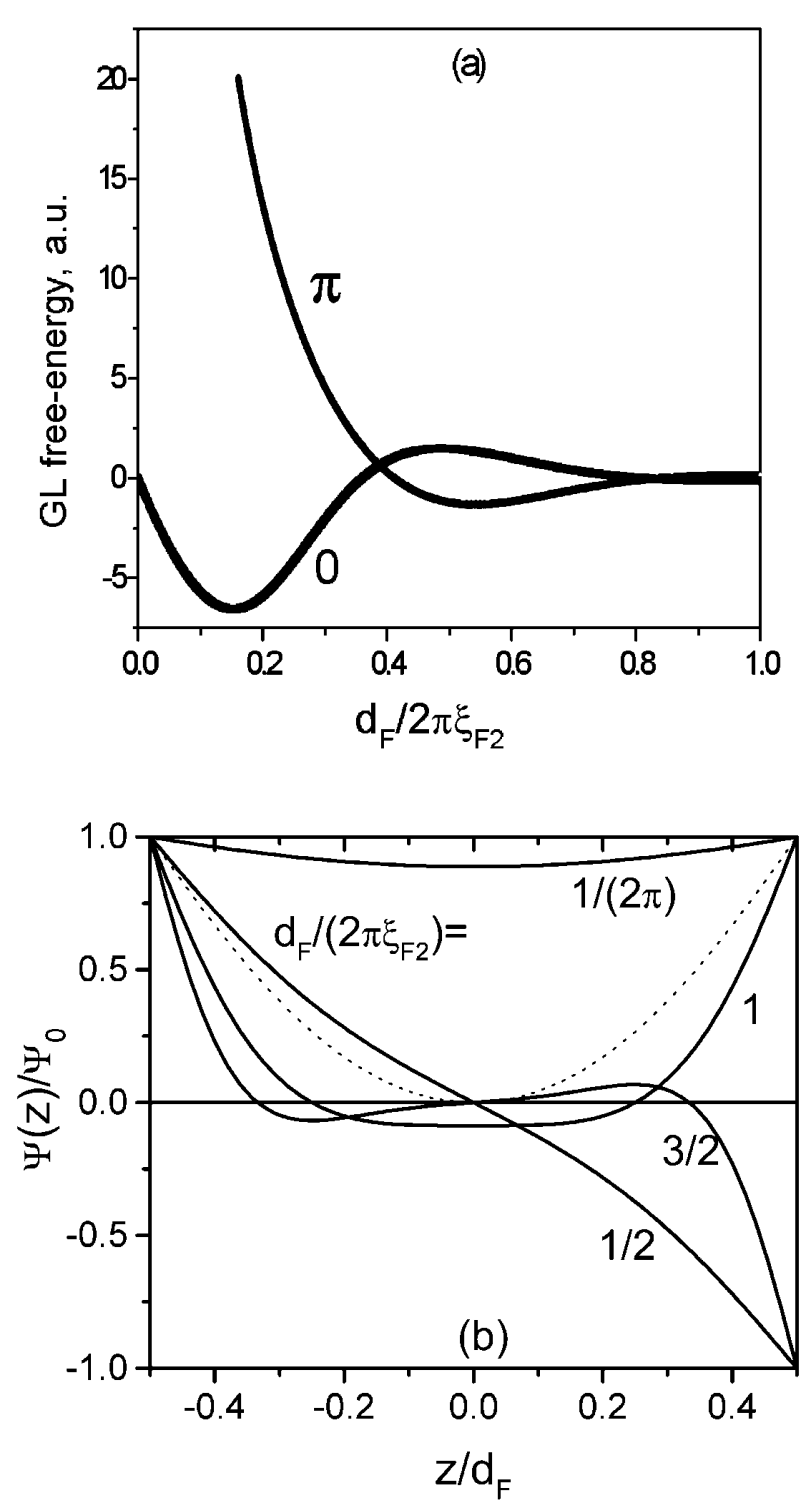

FIG. 1. (a) Calculations of the Ginzburg-Landau (GL) free energy in the $F$ layer for the 0 - and $\pi$-phase states. (b) The spatial distribution of the order parameter in the $F$ layer of the SFS junction calculated for various ratios of $d_{F} /\left(2 \pi \xi_{F 2}\right)$ : for $d_{F} /\left(2 \pi \xi_{F 2}\right)=1 /(2 \pi)$ and 1 the lowest energy corresponds to the 0 phase, while for $d_{F} /\left(2 \pi \xi_{F 2}\right)=1 / 2$ and $3 / 2$ the $\pi$ phase is energetically favorable. Shown for comparison is the 0 phase for $d_{F} /\left(2 \pi \xi_{F 2}\right)=1 / 2$ (dotted line), which has higher energy than the $\pi$ phase.

the order parameter should be written as

$$
\psi(z) \propto e^{-z / \xi_{F}} \propto e^{-z / \xi_{F 1}} e^{-i z / \xi_{F 2}},
$$

with $\xi_{F}$ given by

$$
\xi_{F}=\sqrt{\frac{\hbar D}{2\left(\pi k_{B} T+i E_{\mathrm{ex}}\right)}},
$$

which yields for $\xi_{F 1}$ and $\xi_{F 2}$ :

$$
\xi_{F 1,2}=\sqrt{\frac{\hbar D}{\left[E_{\mathrm{ex}}^{2}+\left(\pi k_{B} T\right)^{2}\right]^{1 / 2} \pm k_{B} T}} .
$$

This reverts to $\xi_{F 1}=\xi_{F 2}$ for $E_{\mathrm{ex}} \gg k_{B} T$ as discussed above, and encountered with classical ferromagnets $(\mathrm{Fe}$, $\mathrm{Co}, \mathrm{Ni}$ ) with $E_{\text {ex }}$ of the order of $1 \mathrm{eV}$. In the case $k_{B} T \approx$ $E_{\text {ex }}$ the decay length $\xi_{F 1}$ increases with decreasing temperature whereas $\xi_{F 2}$ decreases. This is how varying the temperature provides the possibility to cross from a 0 -phase to a $\pi$-phase state [16]. Moreover, a small value for $E_{\text {ex }}$ ensures a large decay length $\xi_{F 1}$, making Josephson SFS sandwiches with homogeneous and continuous ferromagnetic interlayers possible.

The junctions we studied consisted of superconducting $\mathrm{Nb}(\mathrm{S})$ banks with an interlayer of a ferromagnetic $\mathrm{Cu}_{1-x} \mathrm{Ni}_{x}$ alloy $(\mathrm{F})$. The onset of ferromagnetism is around $x=0.44$; above this concentration the Ni magnetic moment increases with about $0.01 \mu_{B} /$ at. $\% \mathrm{Ni}$, which allows precise tuning of the magnetism. An insulating $\mathrm{SiO}$ layer was used between the top electrode and the bottom SF sandwich. The window in this layer determined the junction area of $50 \times 50 \mu \mathrm{m}^{2}$. A schematic sample cross section is given in Fig. 2 (upper panel). Because of the low junction resistance $R_{n} \approx 10^{-5} \Omega$ the transverse transport characteristics were measured by a SQUID picovoltmeter with a sensitivity of $10^{-11} \mathrm{~V}$ in the temperature range of 1.2 to $9 \mathrm{~K}$. Junctions were fabricated with $x$ between 0.40 and 0.57 . Upon crossing to the ferromagnetic regime the junction critical currents dropped sharply but the $I-V$ characteristics and magnetic field dependence $I_{c}(H)$ ( $H$ in the plane of the junction) were still similar to those for standard SNS junctions ( $\mathrm{N}$ is a normal metal). In Fig. 2 (middle panel) $I-V$ data are shown for a junction with $x=0.5, d_{F}=14 \mathrm{~nm}$ at a temperature of $4.2 \mathrm{~K}$. The voltage onset at $I_{c}$ is sharp and well defined. Figure 2 (lower panel) shows that $I_{c}(H)$ for this junction yields the classical "Fraunhofer" pattern. The oscillation period is in reasonable agreement with the cross section of the junction. Note that the central peak is at zero field, even though the alloy is ferromagnetic. This signifies that on average there is no change in the phase difference over the junction along the different directions in the plane of the junction, presumably due to a small-scale magnetic domain structure of the magnetic layer with zero net magnetization. The peak was found shifted when the sample was heated above $T_{c}$ (but below the ferromagnetic transition temperature, $T_{\text {Curie }}$ ) and a small field briefly applied, leading to a finite magnetization. Sometimes the peak was found shifted in zero applied field, probably due to flux trapping in the superconducting banks during cooling down. This could be remedied by reheating and recooling. The starting point for all measurements was a central peak at zero field.

Our main result was obtained for junctions with $\mathrm{Cu}_{0.48} \mathrm{Ni}_{0.52}$ alloys. At this concentration $T_{\text {Curie }}$ is about 20 to $30 \mathrm{~K}$. The resistivity of such alloys at $10 \mathrm{~K}$ is of the order of $50 \mu \Omega \mathrm{cm}$, indicating a mean free path of about $1 \mathrm{~nm}$. The magnetization of the films is in-plane. SQUID magnetometry at $10 \mathrm{~K}$ on single alloy films of thickness 20 to $100 \mathrm{~nm}$, and on $\mathrm{Nb} /$ alloy/Nb trilayers with similar 

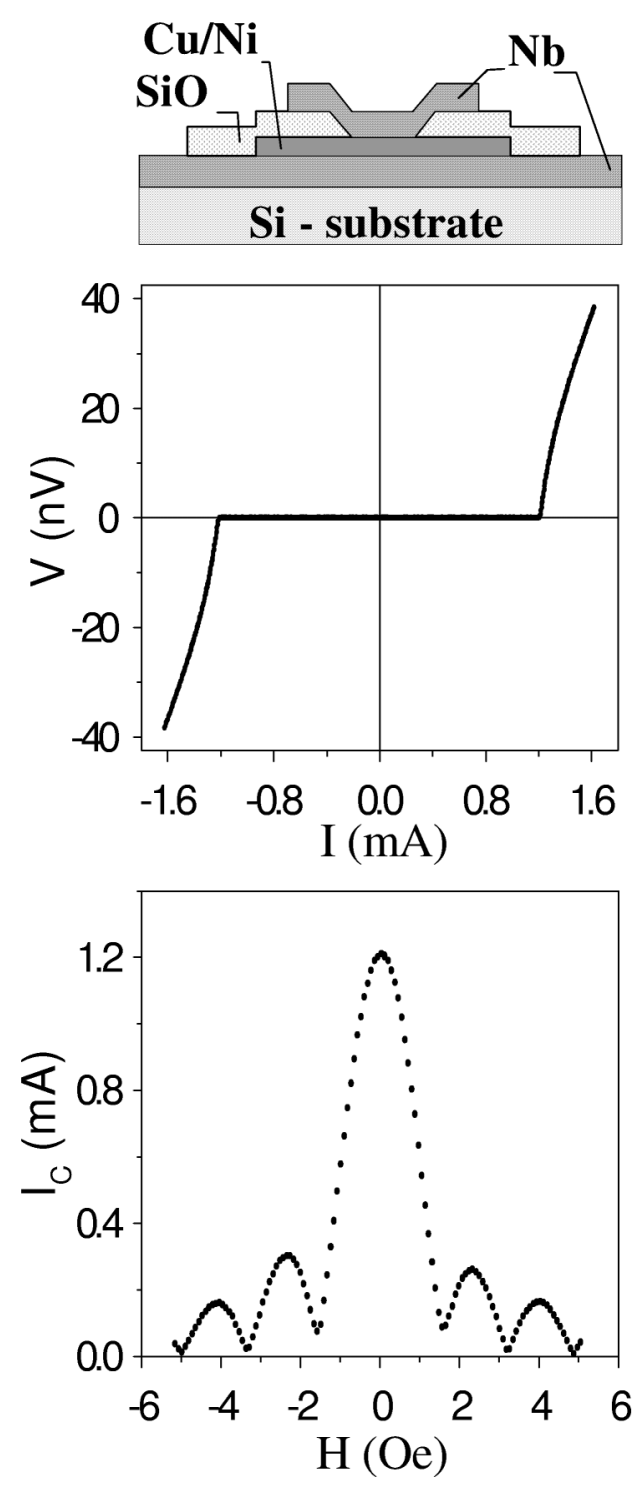

FIG. 2. (Top) Schematic cross section of the sample. (Center) Typical $I-V$ characteristic. (Bottom) Magnetic field dependence of the critical current $I_{c}$ for the junction with $\mathrm{Cu}_{0.5} \mathrm{Ni}_{0.5}$ and $d_{F}=14 \mathrm{~nm}$.

alloy thickness, showed a small hysteresis loop with a coercive field of about $8 \mathrm{mT}$ and a saturation moment of $0.07 \mu_{B} / \mathrm{Ni}$ atom. We found no significant difference between single layers and trilayers, from which we conclude that also in the junctions the alloy layer is ferromagnetic and that a supercurrent can be sustained even through a ferromagnetic layer. Figure 3 shows $I_{c}(T)$ in zero magnetic field for two junctions with $d_{F}=22 \mathrm{~nm}$ [17]. The curve marked $(a)$ shows that $I_{c}$ increases with decreasing temperature, goes through a maximum, returns to zero, and rises again sharply. For all data points, it was ascertained that the zero-field value was the maximum value for $I_{c}$. The curve marked $(b)$ shows the same characteristic behavior although the zero value for $I_{c}$ lies at a different temperature. In this case $I_{c}(H)$ characteristics were measured at three different temperatures to ascertain that $I_{c}$

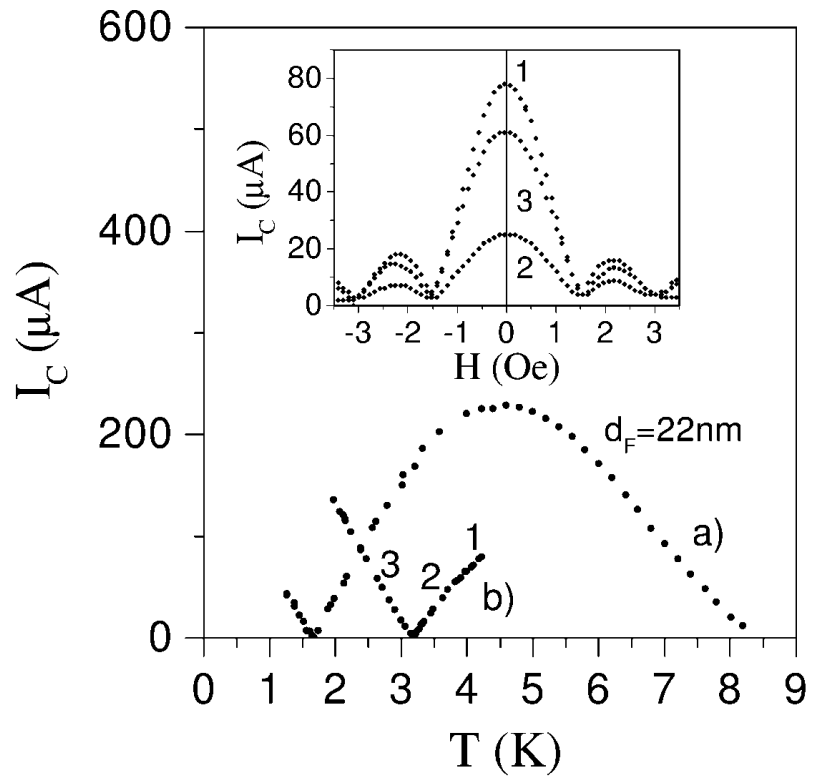

FIG. 3. Critical current $I_{c}$ as a function of temperature $T$ for two junctions with $\mathrm{Cu}_{0.48} \mathrm{Ni}_{0.52}$ and $d_{F}=22 \mathrm{~nm}$ [17]. Inset: $I_{c}$ versus magnetic field $H$ for the temperatures around the crossover to the $\pi$ state as indicated on curve $b$ : (1) $T=4.19 \mathrm{~K}$, (2) $T=3.45 \mathrm{~K}$, (3) $T=2.61 \mathrm{~K}$.

was determined correctly. The data, shown in the inset of Fig. 3, prove that the $I_{c}(T)$ oscillations are not associated with residual magnetic inductance changes which would change the position of the central peak. It is important to realize that the phase difference in zero applied field is uniform in the plane of the junction, either 0 or $\pi$. The Fraunhofer pattern will not shift when the phase turns from 0 to $\pi$, but the zero-field $I_{c}$ goes from positive to negative. In a current-driven experiment, this leads to the sharp cusp observed in $I_{c}(T)$. The $\pi$ state can also be demonstrated by the thickness dependence of the effect. Shown in Fig. 4a is a series of measurements for junctions of different thicknesses in the range 23 to $27 \mathrm{~nm}$. At $23 \mathrm{~nm}$ only positive curvature is visible, an inflection point is observed for $25 \mathrm{~nm}$, a maximum for $26 \mathrm{~nm}$, and the full cusp now at $27 \mathrm{~nm}$. Figure $4 \mathrm{~b}$ shows a set of calculations based on the formalism of the quasiclassical Usadel equations [18], with reasonable parameters for $E_{\mathrm{ex}}$ and $d_{F} / \xi^{*}$, where $\xi^{*}=\left[\hbar D /\left(2 \pi k_{B} T_{c}\right)\right]^{1 / 2}$. They qualitatively demonstrate how the crossover moves into the measurement window upon increasing the $F$-layer thickness. Quantitatively, the thickness dependence in the calculations is much weaker than in the experiments. Parameters such as the spin flip scattering length probably also play a role. Still, the appearance of the crossover is mimicked correctly. If we estimate it around $d_{F} / 2 \pi \xi_{F 2} \approx 0.4-0.5$, it follows that $\xi_{F 2} \approx 10 \mathrm{~nm}$, as expected for the low magnetic moment and justifying the assumption of dirty limit conditions.

A final remark concerns qualitative and quantitative reproducibility. Qualitatively, the cusps can be observed for certain thickness intervals in all sample batches with ferromagnetic layers which are presently fabricated, both for 


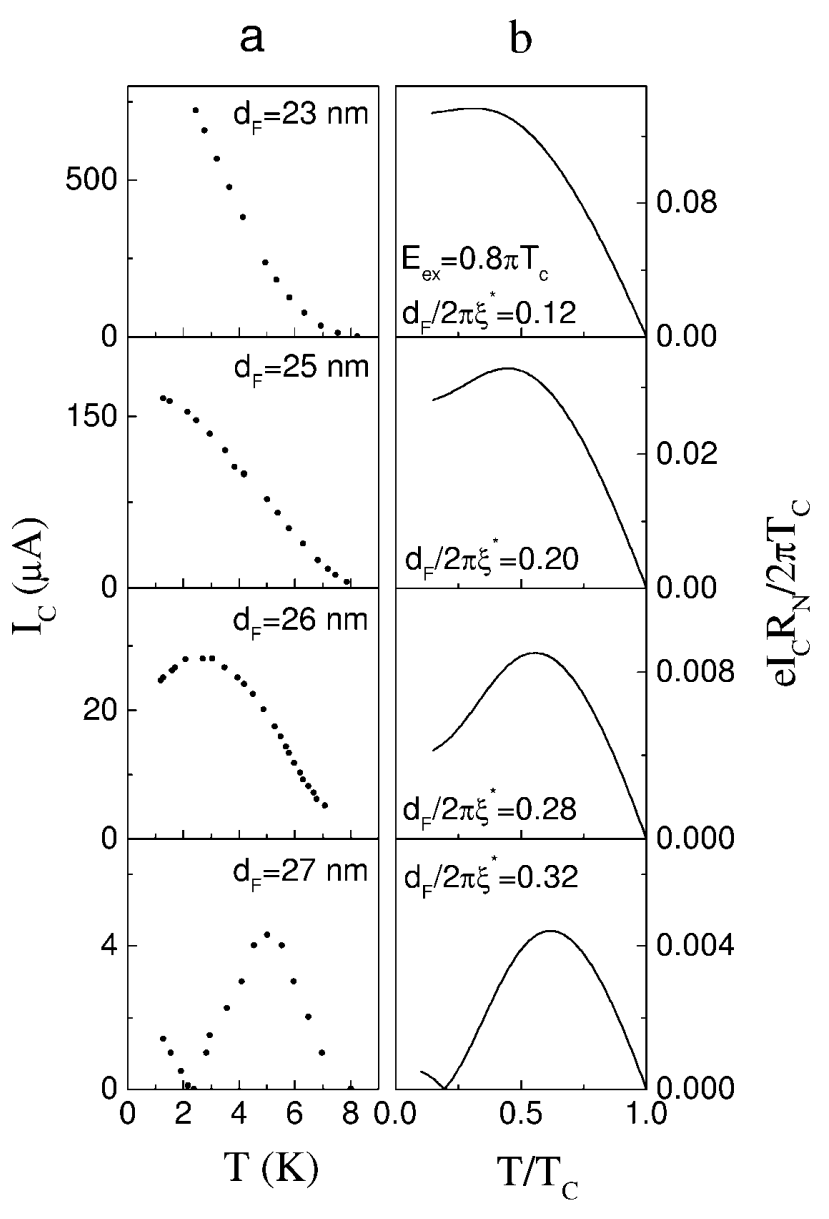

FIG. 4. (a) Critical current $I_{c}$ as a function of temperature for $\mathrm{Cu}_{0.48} \mathrm{Ni}_{0.52}$ junctions with different $F$-layer thicknesses between 23 and $27 \mathrm{~nm}$ as indicated. (b) Model calculations of the temperature dependence of the critical current in a SFS junction for $E_{\mathrm{ex}}=0.8 \pi T_{c}$ and various ratios of $d_{F} / 2 \pi \xi^{*}$, where $\xi^{*}=\sqrt{\hbar D /\left(2 \pi k_{B} T_{c}\right)}$.

concentrations of 52 at. $\% \mathrm{Ni}$ (with $T_{\text {Curie }}$ about $20-30 \mathrm{~K}$ ) and 57 at. $\% \mathrm{Ni}$ (with $T_{\text {Curie }}$ around $100 \mathrm{~K}$ ). Moreover, for higher Ni concentration the crossovers are at lower thickness, reflecting the decrease in $\xi_{F 1, F 2}$. Quantitatively, there are still variations in the values of thickness interval and crossover temperatures, and in the magnitude of the critical current for different batches, even with the same nominal $F$-layer content. Typical batch-to-batch variations are demonstrated in the differences between Figs. 3 and 4. We believe this is due to small variations in the magnetic properties of the $F$ layers. In single films, $T_{\text {Curie }}$ shows a spread of about $10 \mathrm{~K}$; the weak magnetism is apparently sensitive to the details of the preparation procedure.
We thank M. Feigelman for helpful discussion and advice, and N. S. Stepakov for assistance during the experiment. This work was supported by Grant No. 047-005-001 from the Netherlands Organization for Scientific Research (NWO), INTAS-RFBR Grant No. N11459 and RFBR Grant No. N98-02-17045.

[1] A. I. Larkin and Yu. N. Ovchinnikov, Sov. Phys. JETP. 20, 762 (1965) [Zh. Eksp. Teor. Fiz. 47, 1136 (1964)].

[2] P. Fulde and R. A. Ferrel, Phys. Rev. 135, A550 (1964).

[3] L. N. Bulaevskii, V. V. Kuzii, and A. A. Sobyanin, JETP Lett. 25, 290 (1977).

[4] A. I. Buzdin, L. N. Bulaevskii, and S. V. Panjukov, JETP Lett. 35, 178 (1982).

[5] A. I. Buzdin, B. Bujicic, and B. M. Yu. Kupriyanov, Sov. Phys. JETP 74, 124 (1992).

[6] L. B. Ioffe, V. B. Geshkenbein, M. V. Feigel'man, A. L. Fauchere, and G. Blatter, Nature (London) 398, 679 (1999); see also G. Blatter, V. B. Geshkenbein, and L. B. Ioffe, cond-mat/9912163.

[7] J. E. Mooij, T. P. Orlando, L. Levitov, L. Tian, C. H. van der Wal, and S. Lloyd, Science 285, 1036 (1999).

[8] E. A. Demler, G. B. Arnold, and M.R. Beasley, Phys. Rev. B 55, 15174 (1997).

[9] A. V. Andreev, A. I. Buzdin, and R. M. Osgood III, Phys. Rev. B 43, 10124 (1991).

[10] A. I. Buzdin and M. Yu. Kupriyanov, JETP Lett. 52, 487 (1990).

[11] Z. Radović, M. Ledvij, L. Dobrosavljević-Grujić, A. I. Buzdin, and J. R. Clem, Phys. Rev. B 44, 759 (1991).

[12] J. S. Jiang, D. Davidovic, D. H. Reich, and C. L. Chien, Phys. Rev. Lett. 74, 314 (1995).

[13] Th. Mühge, N. N. Garif'yanov, Yu. V. Goryunov, G. G. Khaliullin, L. R. Tagirov, K. Westerholt, I. A. Garifullin, and H. Zabel, Phys. Rev. Lett. 77, 1857 (1996).

[14] J. Aarts, J. M.E. Geers, E. Bruck, A. A. Golubov, and R. Coehoorn, Phys. Rev. B 56, 2779 (1997).

[15] L. Lazar, K. Westerholt, H. Zabel, L. R. Tagirov, Yu. V. Goryunov, N. N. Garif'yanov, and I. A. Garifullin, Phys. Rev. B 61, 3711 (2000).

[16] For a preliminary report on the experimental results, see A. V. Veretennikov et al., Physica (Amsterdam) 284B-288B, 495 (2000); the temperature dependence of the crossover point was recently discussed by T. T. Heikkila, F. K. Wilhelm, and G. Schön, Europhys. Lett. 51, 434 (2000).

[17] It was actually the same junction, but removed after the first measurement between 4.5 and $2 \mathrm{~K}$ and contacted again. We assume that the heating during the contacting procedure led to small changes of the $F$-layer properties.

[18] K. D. Usadel, Phys. Rev. Lett. 25, 507 (1970). 